\title{
Typechecking Top-Down Uniform Unranked Tree Transducers
}

\author{
Wim Martens and Frank Neven \\ University of Limburg \\ E-mail: \{wim.martens,frank.neven\}@luc.ac.be
}

\begin{abstract}
We investigate the typechecking problem for XML queries: statically verifying that every answer to a query conforms to a given output schema, for inputs satisfying a given input schema. As typechecking quickly turns undecidable for query languages capable of testing equality of data values, we return to the limited framework where we abstract XML documents as labeled ordered trees. We focus on simple top-down recursive transformations motivated by XSLT and structural recursion on trees. We parameterize the problem by several restrictions on the transformations (deleting, non-deleting, bounded width) and consider both tree automata and DTDs as output schemas. The complexity of the typechecking problems in this scenario range from PTIME to EXPTIME.
\end{abstract}

\section{Introduction}

The emergence of XML as the likely standard for representing and exchange of data on the Web confirmed the central role of semistructered data. However, it has also marked the return of the schema. In the context of the Web, schemas can be used to validate data exchange. In a typical scenario, a user community agrees on a common schema and on producing only data conforming to that schema. This raises the issue of typechecking: verifying at compile time that every XML document which is the result of a specified query applied to a valid input document, satisfies the output schema [23, 24].

Obviously, typechecking depends on the transformation language and the schema language at hand. As shown by Alon et al. [1,2], when transformation languages have the ability to compare data values, the typechecking problem quickly turns undecidable. Milo, Suciu, and Vianu argued that the capability of most XML transformation languages can be encompassed by $k$-pebble transducers when data values are ignored and XML documents can be abstracted by labeled ordered trees [15]. Further, the authors showed that the typechecking problem in this context is decidable. More precisely, given two types $\tau_{1}$ and $\tau_{2}$, represented by tree automata, and a $k$-pebble transducer $T$, it is decidable whether $T(t) \in \tau_{2}$ for all $t \in \tau_{1}$. Here, $T(t)$ is the tree obtained by running $T$ on input $t$. The complexity, however, is very bad: non-elementary.

In an attempt to lower the complexity, we consider much simpler tree transformations: those defined by deterministic top-down uniform tree transducers 
Table 1. The presented results: the top row of the table shows the representation of the input and output schemas and the left column shows the type of tree transducer.

\begin{tabular}{|l||c|c|c|c|c|}
\hline & NTA & DTA & DTD $($ NFA $)$ & DTD $($ DFA $)$ & DTD $(\mathcal{S} \mathcal{L})$ \\
\hline \hline general & EXPTIME & EXPTIME & EXPTIME & EXPTIME & EXPTIME \\
\hline non-deleting & EXPTIME & EXPTIME & PSPACE & PSPACE & CONP \\
\hline bounded width & EXPTIME & in EXPTIME/PSPACE-hard & PSPACE & PTIME & CONP \\
\hline
\end{tabular}

on unranked trees. Such transformations correspond to structural recursion on trees [5] and to simple top-down XSLT transformations [3,6]. The transducers are called uniform as they cannot distinguish between the order of siblings. In brief, a transformation consists of a top-down traversal of the input tree where every node is replaced by a new tree (possibly the empty tree).

We show that the ability of transducers to delete interior nodes (i.e., replacing them by the empty tree) already renders the typechecking problem EXPTIMEhard for very simple DTDs (e.g. DTDs with deterministic regular expressions on their right hand side). To obtain a lower complexity, for the remainder of the paper, we focus on non-deleting transformations. Our inquiries reveal that the complexity of the typechecking problem of non-deleting transducers is determined by two features: (1) non-determinism in the schema languages; and, (2) unbounded copying of subtrees by the transducer. Only when we disallow both features, we get a PTIME-complexity for the typechecking problem. An overview of our results is given in Table 1. Unless specified otherwise, all complexities are both upper and lower bounds. The top row of the table shows the representation of the input and output schemas and the left column shows the type of tree transducer. NTA and DTA stand for non-deterministic and deterministic tree automata, respectively. Such automata abstract the expressiveness of XML Schema [7]. $\operatorname{DTD}(X)$ stands for DTDs whose right-hand sides consist of regular languages in $X$. The exact definitions are given in section 2 .

Related Work. A problem related to typechecking is type inference $[14,19]$. This problem consists in constructing a tight output schema, given an input schema and a transformation. Of course, solving the type inference problem implies a solution for the typechecking problem: check containment of the inferred schema into the given one. However, characterizing output languages of transformations is quite hard [19].

The transducers considered in the present paper are restricted versions of the ones studied by Maneth and Neven [13]. They already obtained a non-elementary upper bound on the complexity of typechecking (due to the use of monadic second-order logic in the definition of the transducers).

Although the structure of XML documents can be faithfully represented by unranked trees (these are trees without a bound on the number of children of nodes), Milo, Suciu, and Vianu chose to study $k$-pebble transducers over binary trees as there is an immediate encoding of unranked trees into binary ones. The 
top-down variants of $k$-pebble transducers are well-studied on binary trees [11]. However, these results do not aid in the quest to characterize precisely the complexity of typechecking transformations on unranked trees. Indeed, the class of unranked tree transductions can not be captured by ordinary transducers working on the binary encodings. Macro tree transducers can simulate our transducers on the binary encodings $[13,9]$, but as very little is known about their complexity this observation is not of much help. For these reasons, we chose to work directly with unranked tree transducers.

Tozawa considered typechecking w.r.t. tree automata for a fragment of topdown XSLT similar to ours [25]. He adapts the backward type inference technique of [15] and obtains a double exponential time algorithm.

Due to space limitations, we only provide sketches of proofs.

\section{Definitions}

In this section we provide the necessary background on trees, automata, and uniform tree transducers.

\subsection{Trees and Hedges}

We fix a finite alphabet $\Sigma$. The set of unranked $\Sigma$-trees, denoted by $\mathcal{T}_{\Sigma}$, is the smallest set of strings over $\Sigma$ and the parenthesis symbols ')' and '(' such that for $\sigma \in \Sigma$ and $w \in \mathcal{T}_{\Sigma}^{*}, \sigma(w)$ is in $\mathcal{T}_{\Sigma}$. We write $\sigma$ rather than $\sigma()$. Note that there is no a priori bound on the number of children of a node in a $\Sigma$-tree; such trees are therefore unranked. In the following, whenever we say tree, we always mean $\Sigma$-tree. A hedge is a finite sequence of trees. The set of hedges, denoted by $\mathcal{H}_{\Sigma}$, is defined as $\mathcal{T}_{\Sigma}^{*}$.

For every hedge $h \in \mathcal{H}_{\Sigma}$, the set of nodes of $h$, denoted by $\operatorname{Dom}(h)$, is the subset of $\mathbb{N}^{*}$ defined as follows:

- if $h=\varepsilon$, then $\operatorname{Dom}(h)=\emptyset$;

- if $h=t_{1} \cdots t_{n}$ where each $t_{i} \in \mathcal{T}_{\Sigma}$, then $\operatorname{Dom}(h)=\bigcup_{i=1}^{n}\left\{i u \mid u \in \operatorname{Dom}\left(t_{i}\right)\right\}$; and,

- if $h=\sigma(w)$, then $\operatorname{Dom}(h)=\{\varepsilon\} \cup \operatorname{Dom}(w)$.

In the sequel we adopt the following convention: we use $t, t_{1}, t_{2}, \ldots$ to denote trees and $h, h_{1}, h_{2}, \ldots$ to denote hedges. Hence, when we write $h=t_{1} \cdots t_{n}$ we tacitly assume that all $t_{i}$ 's are trees. For every $u \in \operatorname{Dom}(h)$, we denote by $\operatorname{lab}^{h}(u)$ the label of $u$ in $h$. A tree language is a set of trees.

\subsection{DTDs and Tree Automata}

We use extended context-free grammars and tree automata to abstract from DTDs and the various proposals for XML schemas. Further, we parameterize the definition of DTDs by a class of representations $\mathcal{M}$ of regular string languages like, e.g., the class of DFAs or NFAs. For $M \in \mathcal{M}$, we denote by $L(M)$ the set of strings accepted by $M$. 
Definition 1. Let $\mathcal{M}$ be a class of representations of regular string languages over $\Sigma$. A DTD is a tuple $\left(d, s_{d}\right)$ where $d$ is a function that maps $\Sigma$-symbols to elements of $\mathcal{M}$ and $s_{d} \in \Sigma$ is the start symbol. For simplicity, we usually denote $\left(d, s_{d}\right)$ by $d$.

A tree $t$ satisfies $d$ if lab ${ }^{t}(\varepsilon)=s_{d}$ and for every $u \in \operatorname{Dom}(t)$ with $n$ children $\operatorname{lab}^{t}(u 1) \cdots \operatorname{lab}^{t}(u n) \in L\left(d\left(\operatorname{lab}^{t}(u)\right)\right)$. By $L(d)$ we denote the tree language accepted by $d$.

We parameterize DTDs by the formalism used to represent the regular language $\mathcal{M}$. Therefore, we denote by $\operatorname{DTD}(\mathcal{M})$ the class of DTDs where the regular string languages are represented by elements of $\mathcal{M}$. The size of a DTD is the sum of the sizes of the elements of $\mathcal{M}$ used to represent the function $d$.

To define unordered languages we make use of the specification language $\mathcal{S} \mathcal{L}$ inspired by [17] and also used in [1,2]. The syntax of the language is as follows.

Definition 2. For every $\sigma \in \Sigma$ and natural number $i, \sigma^{=i}$ and $\sigma^{\geq i}$ are atomic $\mathcal{S L}$-formulas; true is also an atomic $\mathcal{S L}$-formula. Every atomic $\mathcal{S} \mathcal{L}$-formula is an $\mathcal{S L}$-formula and the negation, conjunction, and disjunction of $\mathcal{S} \mathcal{L}$-formulas are also $\mathcal{S} \mathcal{L}$-formulas.

A string $w$ over $\Sigma$ satisfies an atomic formula $\sigma^{=i}$ if it has exactly $i$ occurrences of $\sigma ; w$ satisfies $\sigma^{\geq i}$ if it has at least $i$ occurrences of $\sigma$. Further, true is satisfied by every string. ${ }^{1}$ Satisfaction of Boolean combinations of atomic formulas is defined in the obvious way. As an example, consider the $\mathcal{S} \mathcal{L}$ formula co-producer $^{\geq 1} \rightarrow$ producer $^{\geq 1}$. This expresses the constraint that a co-producer can only occur when a producer occurs. The size of an $\mathcal{S L}$-formula is the number of symbols that occur in it (every $i$ in $\sigma^{=i}$ or $\sigma^{\geq i}$ is written in binary notation).

We recall the definition of non-deterministic tree automata from [4]. We refer the unfamiliar reader to [16] for a gentle introduction.

Definition 3. A nondeterministic tree automaton (NTA) is a tuple $B=(Q, \Sigma$, $\delta, F)$, where $Q$ is a finite set of states, $F \subseteq Q$ is the set of final states, and $\delta$ is a function $Q \times \Sigma \rightarrow 2^{Q^{*}}$ such that $\delta(q, a)$ is a regular string language over $Q$ for every $a \in \Sigma$ and $q \in Q$.

A run of $B$ on a tree $t$ is a labeling $\lambda: \operatorname{Dom}(t) \rightarrow Q$ such that for every $v \in \operatorname{Dom}(t)$ with $n$ children, $\lambda(v 1) \cdots \lambda(v n) \in \delta\left(\lambda(v), \operatorname{lab}^{t}(v)\right)$. Note that when $v$ has no children, then the criterion reduces to $\varepsilon \in \delta\left(\lambda(v)\right.$, lab $\left.^{t}(v)\right)$. A run is accepting iff the root is labeled with an accepting state, that is, $\lambda(\varepsilon) \in F$. A tree is accepted if there is an accepting run. The set of all accepted trees is denoted by $L(B)$. We extend the definition of $\delta$ to trees and denote this by $\delta^{*}(t)$ : if $t$ consists of only one node labeled with $a$ then $\delta^{*}(t)=\{q \mid \varepsilon \in \delta(q, a)\}$; if $t$ is of the form $a\left(t_{1} \cdots t_{n}\right)$, then $\delta^{*}(t)=\left\{q \mid \exists q_{1} \in \delta^{*}\left(t_{1}\right), \ldots, \exists q_{n} \in \delta^{*}\left(t_{n}\right)\right.$ and $\left.q_{1} \cdots q_{n} \in \delta(q, a)\right\}$. So, $t$ is accepted if $\delta^{*}(t) \cap F \neq \emptyset$.

A tree automaton is bottom-up deterministic if for all $q, q^{\prime} \in Q$ with $q \neq q^{\prime}$ and $a \in \Sigma, \delta(q, a) \cap \delta\left(q^{\prime}, a\right)=\emptyset$. We denote the set of bottom-up deterministic

\footnotetext{
${ }^{1}$ The empty string is obtained by $\bigwedge_{\sigma \in \Sigma} \sigma^{=0}$ and the empty set by $\neg$ true.
} 
NTAs by DTA. A tree automaton is top-down deterministic if for all $q, q^{\prime} \in Q$ with $q \neq q^{\prime}, a \in \Sigma$, and $n \geq 0, \delta(q, a)$ contains at most one string of length $n$.

Like for DTDs, we parameterize NTAs by the formalism used to represent the regular languages in the transition functions $\delta(q, a)$. So, for $\mathcal{M}$ a class of representations of regular languages, we denote by $\operatorname{NTA}(\mathcal{M})$ the class of NTAs where all transition functions are represented by elements of $\mathcal{M}$. The size of an automaton $B$ is then $|Q|+|\Sigma|+\sum_{q, a}|\delta(q, a)|+|F|$. Here, by $|\delta(q, a)|$ we denote the size of the automaton accepting $\delta(q, a)$. Unless explicitly specified otherwise, $\delta(q, a)$ is always represented by an NFA.

Let 2AFA be the class of two-way alternating finite automata [12]. We give without proof the following theorem which will be a useful tool for obtaining upper bounds.

Theorem 1. 1. Emptiness of NTA (NFA) is in PTIME;

2. Emptiness of NTA(2AFA) is in PSPACE.

\subsection{Transducers}

We next define the tree transducers used in this paper. To simplify notation, we restrict to one alphabet. That is, we consider transductions mapping $\Sigma$-trees to $\Sigma$-trees. It is, however, possible to define transductions where the input alphabet differs from the output alphabet.

For a set $Q$, denote by $\mathcal{H}_{\Sigma}(Q)$ (resp. $\left.\mathcal{T}_{\Sigma}(Q)\right)$ the set of $\Sigma$-hedges (resp. trees) where leaf nodes can be labeled with elements from $Q$.

Definition 4. A uniform tree transducer is a tuple $\left(Q, \Sigma, q_{0}, R\right)$, where $Q$ is a finite set of states, $\Sigma$ is the input and output alphabet, $q_{0} \in Q$ is the initial state, and $R$ is a finite set of rules of the form $(q, a) \rightarrow h$, where $a \in \Sigma, q \in Q$, and $h \in \mathcal{H}_{\Sigma}(Q)$. When $q=q_{0}, h$ is restricted to $\mathcal{T}_{\Sigma}(Q) \backslash Q$.

The restriction on rules with the initial state ensures that the output is always a tree rather than a hedge. For the remainder of this paper, when we say tree transducer, we always mean uniform tree transducer.

Example 1. Let $T=(Q, \Sigma, p, R)$ where $Q=\{p, q\}$ and $R$ contains the rules

$$
\begin{aligned}
(p, a) \rightarrow d(e) & (p, b) \rightarrow c(q p) \\
(q, a) \rightarrow c q & (q, b) \rightarrow d(q)
\end{aligned} .
$$

Our definition of tree transducers corresponds to structural recursion [5] and a fragment of top-down XSLT. For instance, the XSLT program equivalent to the above transducer is given in Figure 1 (we assume the program is started in mode $p$ ).

The translation defined by $T=\left(Q, \Sigma, q_{0}, R\right)$ on a tree $t$ in state $q$, denoted by $T^{q}(t)$, is inductively defined as follows: if $t=\varepsilon$ then $T^{q}(t):=\varepsilon$; if $t=a\left(t_{1} \cdots t_{n}\right)$ and there is a rule $(q, a) \rightarrow h \in R$ then $T^{q}(t)$ is obtained from $h$ by replacing 
Definition 5. A tree transducer $T$ typechecks w.r.t. to an input tree language $S_{\text {in }}$ and an output tree language $S_{\text {out }}$, if $T(t) \in S_{\text {out }}$ for every $t \in S_{\text {in }}$.

We parameterize the typechecking problem by the kind of tree transducers and tree languages we allow. Let $\mathcal{T}$ be a class of transducers and $\mathcal{S}$ be a class of tree languages. Then $\mathrm{TC}[\mathcal{T}, \mathcal{S}]$ denotes the typechecking problem where $T \in \mathcal{T}$ and $S_{\mathrm{in}}, S_{\text {out }} \in \mathcal{S}$. The size of the input of the typechecking problem is the sum of the sizes of the input and output schema and the tree transducer.

A transducer $T$ has width $k$ if there are at most $k$ occurrences of states in every rhs of $T$. By $\mathcal{B W}_{k}$ we denote the class of transducers of width $k$. A transducer is non-deleting if no states occur at the top-level of a rhs. We denote by $\mathcal{T}_{g}$ the class of all transducers and by $\mathcal{T}_{n d}$ the class of non-deleting transducers. For a class of representations of regular string languages $\mathcal{M}$, we write $\operatorname{TC}[\mathcal{T}, \mathcal{M}]$ rather than $\operatorname{TC}[\mathcal{T}, \operatorname{DTD}(\mathcal{M})]$.

\section{The General Case}

When we do not restrict our transducers in any way, the typechecking problem is in EXPTIME and is EXPTIME-hard for even the simplest DTDs: those where the right-hand sides are specified with $\mathcal{S L}$-formulas or with DFAs. The main reason is that the deleting states allow the transducer to simulate deterministic top-down tree automata in such a way that the transducer produces no output besides acceptance information. In this way, even a very simple DTD can check whether the output was rejected or not. By copying the input several times, we can execute several deterministic tree automata in parallel. These are all the ingredients we need for a reduction from non-emptiness of the intersection of an arbitrary number of deterministic tree automata which is known to be EXPTIME-hard.

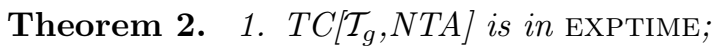

2. $T C\left[\mathcal{T}_{g}, \mathcal{S} \mathcal{L}\right]$ is EXPTIME-hard;

3. $T C\left[\mathcal{T}_{g}, D F A\right]$ is EXPTIME-hard.

Proof. (Sketch) (1) Let $T=\left(Q_{T}, \Sigma, q_{T}^{0}, R_{T}\right)$ be a transducer and let $A_{\text {in }}$ and $A_{\text {out }}=\left(Q_{A}, \Sigma, \delta_{A}, F_{A}\right)$ be two NTAs representing the input and output schema, respectively. We next describe a non-deleting transducer $S$ and an NTA $B_{\text {out }}$ which can be constructed in LOGSPACE, such that $T$ typechecks w.r.t. $A_{\text {in }}$ and $A_{\text {out }}$ iff $S$ typechecks w.r.t. $A_{\text {in }}$ and $B_{\text {out }}$. From Theorem 3(1) it then follows that $\mathrm{TC}\left[\mathcal{T}_{g}, \mathrm{NTA}\right]$ is in EXPTIME.

Intuitively, $S$ puts a \# whenever $T$ would process a deleting state. For instance, the rule $(q, a) \rightarrow c q$ is replaced by $(q, a) \rightarrow c \#(q)$. We introduce some notation to characterize the behavior of $B_{\text {out }}$. Define the \#-eliminating function $\gamma$ as follows: $\gamma(\sigma(h))$ is $\gamma(h)$ when $\sigma=\#$ and $\sigma(\gamma(h))$ otherwise; further, $\gamma\left(t_{1} \cdots t_{n}\right):=\gamma\left(t_{1}\right) \cdots \gamma\left(t_{n}\right)$. Then, clearly, for all $t \in \mathcal{T}_{\Sigma}, T(t)=\gamma(S(t))$. $B_{\text {out }}$ then accepts a tree $t$ iff $\gamma(t) \in L\left(A_{\text {out }}\right)$. 


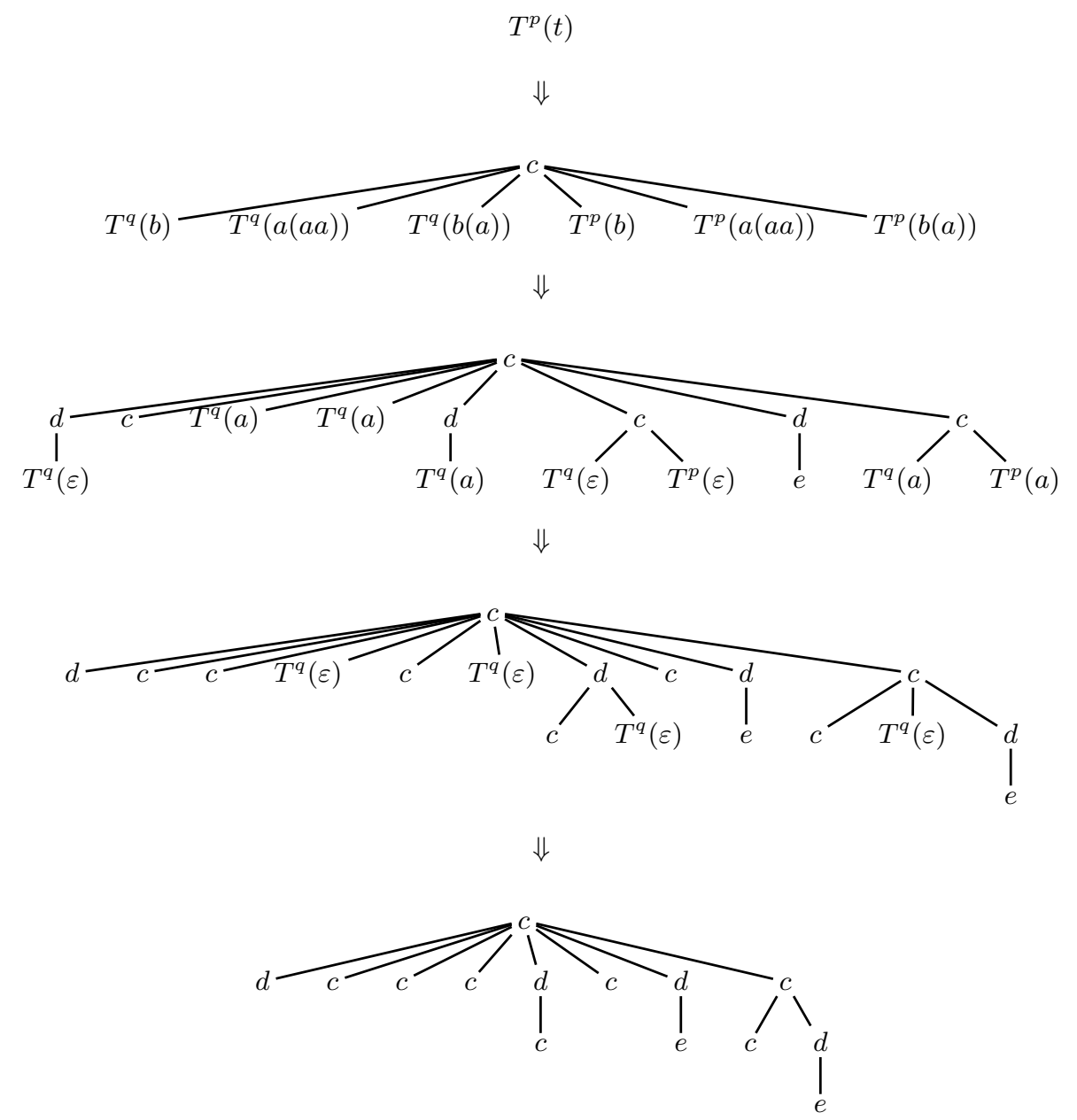

Fig. 2. The translation of $t=b(b a(a a) b(a))$ by the transducer $T$ of Example 1 . 
(2) We use a reduction from the intersection problem of deterministic binary top-down tree automata $A_{i}(i=1 \ldots n)$, which is known to be hard for ExPTIME [21]. The problem is stated as follows, given deterministic binary top-down automata $A_{1}, \ldots, A_{n}$, is $\bigcap_{i=1}^{n} L\left(A_{i}\right)=\emptyset$ ? We define a transducer $T$ and two DTDs $d_{\text {in }}$ and $d_{\text {out }}$ such that $\bigcap_{i=1}^{n} L\left(A_{i}\right) \neq \emptyset$ iff $T$ does not typecheck w.r.t. $d_{\text {in }}$ and $d_{\text {out }}$. In the construction, we exploit the copying power of transducers to make $n$ copies of the input tree: one for each $A_{i}$. By using deleting states, we can execute each $A_{i}$ on its copy of the input tree without producing output. When an $A_{i}$ does not accept, we output an error symbol under the root of the output tree. The output DTD should then only check that an error symbol always appears.

The proof of (3) is similar to the one for (2).

\section{Non-deleting Transformations}

In an attempt to lower the complexity, we consider, in the present section, nondeleting transformations w.r.t. various schema formalisms. We observe that when schemas are represented by tree automata, the complexity remains EXPTIMEhard. When tree languages are represented by DTDs, the complexity of the typechecking problem drops to PSPACE and is hard for PSPACE even when righthand sides of rules are represented by DFAs. The main reason for this is that the tree transducers can still make an unbounded number of copies of the input tree. This allows to simulate in parallel an unbounded number of DFAs and makes it possible to reduce the intersection emptiness problem of DFAs to the typechecking problem. In the next section, we therefore constrain this copying power. In summary, we prove the following results:

Theorem 3. 1. TC $\left[\mathcal{T}_{n d}, N T A\right]$ is EXPTIME-complete;

2. $T C\left[\mathcal{I}_{n d}, D T A\right]$ is EXPTIME-complete;

3. $T C\left[\mathcal{T}_{n d}, N F A\right]$ is PSPACE-complete;

4. $T C\left[\mathcal{T}_{n d}, D F A\right]$ is PSPACE-complete;

5. $T C\left[\mathcal{T}_{n d}, \mathcal{S} \mathcal{L}\right]$ is CONP-complete.

\subsection{Tree Automata}

Consider the case where the input and output schemas are represented by NTA(NFA)s. One way to obtain an appropriate typechecking algorithm, would be to build a composite automaton $A_{\text {co }}$ that on input $t$, runs $A_{\text {out }}$ on $T(t)$ without actually constructing $T(t)$. The given instance typechecks iff $L\left(A_{\text {in }}\right) \subseteq L\left(A_{\text {co }}\right)$. However, constructing $A_{\text {co }}$ would lead to an exponential blowup because the state set of $A_{\text {co }}$ would be $2^{Q_{T}} \times 2^{Q_{\text {out }}}$. Since $A_{\text {co }}$ is nondeterministic because $A_{\text {out }}$ is nondeterministic, solving the inclusion problem on this instance would lead to a double exponential time algorithm. Thus, to show that $\mathrm{TC}\left[\mathcal{T}_{n d}, \mathrm{NTA}\right]$ can be solved in ExPTIME, we need a slightly more sophisticated approach.

Theorem 3(1). TC $\left[\mathcal{T}_{n d}, N T A\right]$ is EXPTIME-complete. 
Proof. (Sketch) Hardness is immediate as containment of NTAs is already hard for EXPTIME [20]. We, therefore, only prove membership in EXPTIME. The proof is similar in spirit to a proof in [18], which shows that containment of Query Automata is in ExPTIME. Let $T=\left(Q_{T}, \Sigma, q_{T}^{0}, R_{T}\right)$ be a non-deleting tree transducer and let $A_{\text {in }}=\left(Q_{\text {in }}, \Sigma, \delta_{\text {in }}, F_{\text {in }}\right)$ and $A_{\text {out }}=\left(Q_{\text {out }}, \Sigma, \delta_{\text {out }}, F_{\text {out }}\right)$ be the NTAs representing the input and output schema, respectively.

For ease of exposition, we restrict hedges in the rhs of $T$ to be trees. In brief, our algorithm computes the set

$$
\begin{aligned}
P=\left\{(S, f) \mid S \subseteq Q_{\text {in }}, f: Q_{T} \rightarrow 2^{Q_{\text {out }}}, \exists t\right. \text { such that } \\
\\
\left.\qquad S=\delta_{\text {in }}^{*}(t) \text { and } \forall q \in Q_{T}, f(q)=\delta_{\text {out }}^{*}\left(T^{q}(t)\right)\right\} .
\end{aligned}
$$

Intuitively, in the definition of $P, t$ can be seen as a witness of $(S, f)$. That is, $S$ is the set of states reachable by $A_{\text {in }}$ at the root of $t$, while for each state $q$ of the transducer, $f(q)$ is the set of states reachable by $A_{\text {out }}$ at the root of $T^{q}(t)$ (recall that this is the translation of $t$ started in state $q$ ). So, the given instance does not typecheck iff there exists an $(S, f) \in P$ such that $F_{i n} \cap S \neq \emptyset$ and $F_{\text {out }} \cap f\left(q_{T}^{0}\right)=\emptyset$. In Figure 3, an algorithm for computing $P$ is depicted. By $\operatorname{rhs}(q, a)\left[p \leftarrow f_{1}(p) \cdots f_{n}(p) \mid p \in Q_{T}\right]$, we denote the tree obtained from $\operatorname{rhs}(q, a)$ by replacing every occurrence of a state $p$ by the sequence $f_{1}(p) \cdots f_{n}(p)$. For $c \in\{$ in, out $\}, \delta_{c}^{*}: \mathcal{T}_{\Sigma}\left(2^{Q_{c}}\right) \rightarrow 2^{Q_{c}}$ is the transition function extended to trees in $\mathcal{T}_{\Sigma}\left(2^{Q_{c}}\right)$. To be precise, for $a \in \Sigma, \delta_{c}^{*}(a):=\left\{q \mid \varepsilon \in \delta_{c}(q, a)\right\}$; for $P \subseteq Q_{c}$, $\delta_{c}^{*}(P):=P$; and, $\delta_{c}^{*}\left(a\left(t_{1} \cdots t_{n}\right)\right):=\left\{q \mid \exists q_{i} \in \delta_{c}^{*}\left(t_{i}\right): q_{1} \cdots q_{n} \in \delta_{c}^{*}(q, a)\right\}$. The correctness of the algorithm follows from the following lemma which can be easily proved by induction.

Lemma 1. A pair $(S, f)$ has a witness tree of depth $i$ iff $(S, f) \in P_{i}$.

It remains to show that the algorithm is in Exptime. The set $P_{1}$ can be computed in time polynomial in the sizes of $A_{\text {in }}, A_{\text {out }}$, and $T$. As $P_{i} \subseteq P_{i+1}$ for all $i$, the loop can only make an exponential number of iterations. It, hence, suffices to show that each iteration can be done in ExPTIME. Actually, we argue that it can be checked in PSPACE whether a tuple $(S, f) \in P_{i}$. Indeed, the question whether there are $\left(S_{1}, f_{1}\right) \cdots\left(S_{n}, f_{n}\right) \in P_{i-1}^{*}$ can be reduced to the emptiness test of a 2AFA $A$ which works on strings over the alphabet $P_{i-1}$. On input $\left(S_{1}, f_{1}\right) \cdots\left(S_{n}, f_{n}\right)$ the $2 \mathrm{AFA} A$ operates as follows: for every $p \in S$ it checks whether there are $r_{i} \in S_{i}$ such that $r_{1} \ldots r_{n} \in \delta(p, a)$. This can be done by $|S|$ traversals through the input string. Next, $A$ checks for every $q \in Q_{i n} \backslash S$ whether for all $r_{i} \in S_{i}, r_{1} \ldots r_{n} \notin \delta(q, a)$. This can be done by $\left|Q_{i n} \backslash S\right|$ traversals through the input string while using alternation. In a similar way $f(q)$ is checked. The automaton $A$ is exponential in the input. However, we can construct $A$ on the fly when executing the PSPACE algorithm for non-emptiness. The latter algorithm is an adaptation of the technique used by Vardi [26]. As there are only exponentially many tuples $(S, f)$, the overall algorithm is in EXPTIME.

In the remainder of this section, we examine what happens when tree automata are restricted to be deterministic. From the above result, it is immediate 


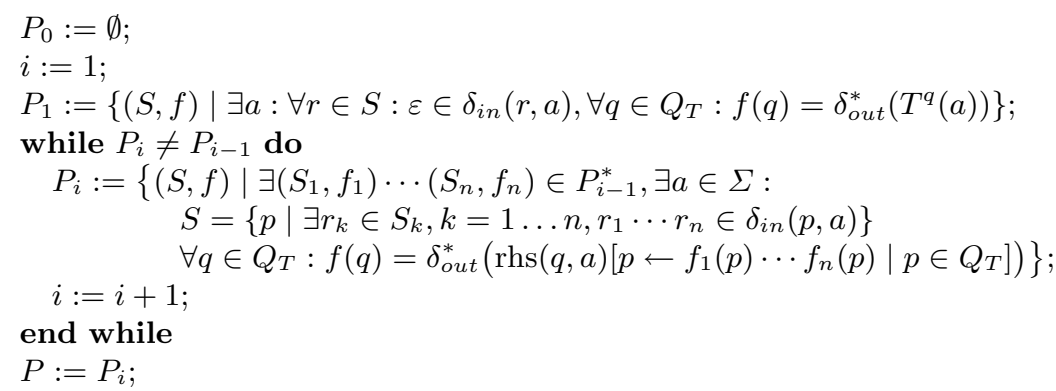

Fig. 3. The algorithm of Theorem 3(1) computing $P$.

that $\mathrm{TC}\left[\mathcal{T}_{n d}, \mathrm{DTA}\right]$ is in ExPTIME. To show that it is hard, we can use a reduction from the intersection problem of deterministic binary top-down tree automata like in the proof of Theorem 2(2). The reduction is almost identical to the one in Theorem 2(2): $A_{\text {in }}$ defines the same set of trees as $d_{\text {in }}$ does with the exception that $A_{\text {in }}$ enforces an ordering of the children. The transducer in the proof of Theorem 2(2) starts the in parallel simulation of the $n$ automata, but then, using deleting states, delays the output until it has reached the leaves of the input tree. In the present setting, we can not use deleting states. Instead, we copy the input tree and attach error-symbols to the leaves when an automaton rejects. The output automaton then checks whether at least one error occurred. We obtain the following theorem.

Theorem 3(2) TC $\left[\mathcal{T}_{n d}, D T A\right]$ is EXPTIME-complete.

\subsection{DTDs}

When we consider DTDs as input schemas the complexity drops to PSPACE and CONP.

Theorem 3(3) TC $\left[\mathcal{T}_{n d}, N F A\right]$ is PSPACE-complete.

Proof. (Sketch) The hardness result is immediate as containment of regular expressions is known to be PSPACE-hard [22]. For the other direction, let $T$ be a non-deleting tree transducer. Let $d_{\text {in }}$ and $d_{\text {out }}$ be the input and output DTDs, respectively. We construct an $\mathrm{NTA}(2 \mathrm{AFA}) B$ such that $L(B)=\left\{t \in L\left(d_{\text {in }}\right) \mid\right.$ $\left.T(t) \notin d_{\text {out }}\right\}$. Moreover, the size of $B$ is polynomial in the size of $T, d_{\text {in }}$, and $d_{\text {out }}$. Thus, $L(B)=\emptyset$ iff $T$ typechecks w.r.t. $d_{\text {in }}$ and $d_{\text {out }}$. By Theorem $1(2)$, the latter is in PSPACE. To explain the operation of the automaton we introduce the following notion: let $q$ be a state of $T$ and $a \in \Sigma$ then define $q(a):=z$ where $z$ is the concatenation of the labels of the top most nodes of $\operatorname{rhs}(q, a)$. For a string $w:=a_{1} \cdots a_{n}$, we define $p(w):=p\left(a_{1}\right) \cdots p\left(a_{n}\right)$. Intuitively, the automaton $B$ now works bottom-up as follows: (1) $B$ checks that $t \in L\left(d_{\text {in }}\right)$; $(2)$ at the same time, $B$ guesses a node $v$ labeled $\sigma$ with $n$ children and picks a state $q$ in which 
$v$ is processed: $B$ then accepts if $h$ does not satisfy $d_{\text {out }}$, where $h$ is obtained from $\operatorname{rhs}(\sigma, q)$ by replacing every state $p$ by $p\left(\operatorname{lab}^{t}(u 1) \cdots \operatorname{lab}^{t}(u n)\right)$. As $d_{\text {out }}$ is specified by NFAs and we have to check that $d_{\text {out }}$ is not satisfied, we need alternation to specify the transition function of $B$. Additionally, as $T$ can copy its input, we need two-way automata.

Formally, let $T=\left(Q_{T}, \Sigma, q_{0}^{T}, \delta_{T}\right)$. Define $B=\left(Q^{B}, \Sigma, F^{B}, \delta^{B}\right)$ as follows. The set of states $Q^{B}$ is the union of the following sets: $\Sigma,\left\{(\sigma, q) \mid q \in Q_{T}, \sigma \in\right.$ $\Sigma\}$, and $\left\{(\sigma, q\right.$, check $\left.) \mid q \in Q_{T}, \sigma \in \Sigma\right\}$. If there is an accepting run on a tree $t$, then a node $v$ labeled with a state of the form $\sigma,(\sigma, q),(\sigma, q$, check) has the following meaning:

$\sigma$ : the current node is labeled with $\sigma$ and the subtree rooted at this node satisfies $d_{\text {in. }}$.

$(\sigma, q)$ : same as in previous case with the following two additions: (1) $v$ is processed by $T$ in state $q$; and, (2) a descendant of $v$ will produce a tree that will not satisfy $d_{\text {out }}$.

$(\sigma, q$, check): same as the previous case only now $v$ itself will produce a tree that does not satisfy $d_{\text {out }}$.

The set of final states is defined as follows: $F^{B}:=\left\{\left(\sigma, q_{0}^{T}\right) \mid \sigma \in \Sigma\right\}$. The transition function is defined as follows:

1. $\delta^{B}(a, b)=\delta^{B}((a, q), b)=\delta^{B}((a, q$, check $), b)=\emptyset$ for all $a \neq b$;

2. $\delta^{B}(a, a)=d_{\text {in }}(a)$ and

$\delta^{B}((a, q), a)=\left\{\Sigma^{*}(b, p) \Sigma^{*}+\Sigma^{*}(b, p\right.$, check $) \Sigma^{*} \mid p \operatorname{occurs}$ in $\left.\operatorname{rhs}(q, a), b \in \Sigma\right\}$,

for all $a \in \Sigma$ and $q \in Q_{T}$.

3. Finally, $\delta^{B}((a, q$, check $), a)=\left\{a_{1} \cdots a_{n} \mid h \notin L\left(d_{\text {out }}\right)\right.$ and $\left.a_{1} \cdots a_{n} \in d_{\text {in }}(a)\right\}$. Here, $h$ is obtained from $\operatorname{rhs}(q, a)$ by replacing every $q$ by $q\left(a_{1} \cdots a_{n}\right)$.

We are left with the proof that $\delta^{B}((a, q$, check $), a)$ can be computed by a 2AFA $A$ with only a polynomial blowup. Before we define $A$, we define some other automata. First, for every $b \in \Sigma$, let $A_{b}$ be the NFA accepting $d_{\text {out }}(b)$.

For every $v$ in $\operatorname{rhs}(q, a)$, let $w$ be the largest string in $\left(\Sigma \cup Q_{T}\right)^{*}$ such that $\operatorname{lab}^{h}(v)(w)$ is a subtree rooted at $v$ in $h$. Define the 2NFA $B_{v}$ as follows: suppose $w$ is of the form $z_{0} p_{1} z_{1} \cdots p_{\ell} z_{\ell}$, then $a_{1} \cdots a_{n} \in L\left(B_{v}\right)$ if and only if $z_{0} p_{1}\left(a_{1} \cdots a_{n}\right) z_{1} \cdots p_{\ell}\left(a_{1} \cdots a_{n}\right) z_{\ell} \in L\left(A_{\mathrm{lab}^{h}(v)}\right)$. As $w$ is fixed, $B_{v}$ can recognize this language by reading $a_{1} \cdots a_{n} \ell$ times while simulating $A_{\mathrm{lab}^{h}(v)}$. Intuitively, the automaton simulates $A_{\mathrm{lab}^{h}(v)}$ on $z_{i-1} p_{i}\left(a_{1} \cdots a_{n}\right)$ on the $i$ th pass.

It remains to describe the construction of $A$. To this end, let $A_{i n}^{a}$ be the NFA such that $d_{\text {in }}(a)=L\left(A_{i n}^{a}\right)$. On input $a_{1} \cdots a_{n}$, $A$ first checks whether $a_{1} \cdots a_{n} \in L\left(A_{i n}^{a}\right)$ by simulating $A_{i n}^{a}$. After this, $A$ goes back to the beginning of the input string, guesses an internal node $u$ in $\operatorname{rhs}(q, a)$ and simulates the negation of $B_{u}$. As $B_{u}$ is a $2 \mathrm{NFA}, A$ is a $2 \mathrm{AFA}$.

The intersection problem of deterministic finite automata is known to be PSPACE-hard [10] and is easily reduced to $\mathrm{TC}\left[\mathcal{T}_{n d}, \mathrm{DFA}\right]$. This implies the following result: 
Theorem 3(4) TC $\left[\mathcal{T}_{n d}, D F A\right]$ is PSPACE-complete.

Using $\mathcal{S} \mathcal{L}$-expressions to define right-hand sides of DTDs reduces the complexity of typechecking to CONP.

Theorem 3(5) $T C\left[\mathcal{T}_{n d}, \mathcal{S} \mathcal{L}\right]$ is CONP-complete.

Proof. (Sketch) First, we prove the hardness result. Let $\phi$ be a SAT-formula and let $v_{1}, \ldots, v_{n}$ be the variables occurring in $\phi$. We define the typechecking instance as follows. $\Sigma=\left\{\sigma_{1}, \ldots, \sigma_{n}\right\}$. We only define $d_{\text {in }}$ and $d_{\text {out }}$ for $\sigma_{1}$, since this is all we require. $d_{\text {in }}\left(\sigma_{1}\right)=\phi^{\prime}$, where $\phi^{\prime}$ is the formula $\phi$ with every occurrence of $v_{i}$ replaced by $\sigma_{i}^{=1}$ for $i=1 \ldots n$. The transducer $T$ is the identity, and $d_{\text {out }}\left(\sigma_{1}\right)=\emptyset$. Hence, this instance typechecks iff $\phi$ is not satisfiable.

To prove the upper bound, let $T=\left(Q_{T}, \Sigma, q_{T}^{0}, R_{T}\right)$ and let $\left(d_{\text {in }}, s_{\text {in }}\right)$ and $\left(d_{\text {out }}, s_{\text {out }}\right)$ be the input and output DTD respectively. We describe an NP algorithm that accepts iff the given instance does not typecheck.

We introduce some notation. For a $\operatorname{DTD}\left(d, s_{d}\right)$ and $\sigma \in \Sigma$, we denote by $d^{\sigma}$ the DTD $d$ with start symbol $\sigma$, that is, $(d, \sigma)$. Let $k$ be the largest number occurring in an $\mathcal{S} \mathcal{L}$-formula in $d_{\text {in }}$. Set $r=(k+1) \times|\Sigma|$.

The algorithm consists of three main parts:

1. First, we sequentially guess a subset $L$ of the derivable symbols $\{b \in \Sigma \mid$ $\left.L\left(d_{\text {in }}^{b}\right) \neq \emptyset\right\}$.

2. Next, we guess a path of a tree in $d_{\text {in }}$. In particular, we guess a sequence of pairs $\left(a_{i}, q_{i}\right) \in L \times Q_{T}, i=0, \ldots, m$, with $m \leq|\Sigma| \times\left|Q_{T}\right|$, such that

(a) $a_{0}=s_{\text {in }}$ and $q_{0}=q_{T}^{0}$;

(b) $\exists t, \exists u \in \operatorname{Dom}(t)$ such that $a_{0} \cdots a_{m}$ is the concatenation of the labels of the nodes on the path from the root to $u$; and,

(c) $\forall i=1, \ldots, m: T$ visits $a_{i}$ in state $q_{i}$.

3. Finally, we guess a string $w \in L^{*}$ of length at most $r$ such that $T^{q_{m}}\left(a_{m}(w)\right) \notin$ $L\left(d_{\text {out }}^{\sigma}\right)$ with $\sigma$ the root symbol of $T^{q_{m}}\left(a_{m}(w)\right)$.

All guesses can be done at once and can be checked by a polynomial verifier. This completes the description of the algorithm.

\section{Transducers of Bounded Width}

When we put a bound on the width (or copying power, recall the discussion at the end of sections 2.3 and 2.4) of transducers we get a PTIME algorithm for typechecking when the right-hand sides of DTDs are represented by DFAs. All other results have the same complexity as in the case of unrestricted copying.

Theorem 4. 1. TC $\left[\mathcal{B} \mathcal{W}_{k}, N T A\right]$ is EXPTIME-complete;

2. $T C\left[\mathcal{B} \mathcal{W}_{k}, R E\right]$ is PSPACE-complete;

3. $T C\left[\mathcal{B} \mathcal{W}_{k}, D F A\right]$ is PTIME-complete;

4. $T C\left[\mathcal{B} \mathcal{W}_{k}, \mathcal{S L}\right]$ is CONP-complete. 
The lower bounds of (1), (2), and (4) follow immediately from the construction in the proofs of Theorem 3(1), (3), and (5).

Theorem 4(3) TC $\left[\mathcal{B} \mathcal{W}_{k}, D F A\right]$ is PTIME-complete.

Proof. (Sketch) In the proof of Theorem $3(3)$, TC $\left[\mathcal{T}_{n d}, \mathrm{NFA}\right]$ is reduced to the emptiness of NTA(2AFA)s. Alternation was needed to express negation of NFAs; two-wayness was needed because $T$ could make arbitrary copies of the input tree. However, when transducers can make only a bounded number of copies and DFAs are used, TC $\left[\mathcal{B W}_{k}, \mathrm{DFA}\right]$ can be LOGSPACE-reduced to emptiness of NTA(NFA)s. From Theorem $1(1)$, it then follows that $\mathrm{TC}\left[\mathcal{B} \mathcal{W}_{k}, \mathrm{DFA}\right]$ is in PTIME. A PTIME lower bound is obtained by a reduction from PATH SYSTEMS [8].

\section{Conclusion}

Motivated by structural recursion and XSLT, we studied typechecking for topdown XML transformers in the presence of both DTDs and tree automata. In this setting the complexity of the typechecking problem ranges from PTIME to EXPTIME. In particular, the PTIME algorithm is obtained by restricting to nondeleting tree transducers of bounded width and DTD(DFA)s. The main open question for future research is how these restrictions can be relaxed while still having a PTIME algorithm. Another question we left open is the exact complexity of TC $\left[\mathcal{B W}_{k}, \mathrm{DTA}\right]$ which is in EXPTIME and PSPACE-hard.

\section{References}

1. N. Alon, T. Milo, F. Neven, D. Suciu, and V. Vianu. Typechecking XML views of relational databases. In Proc. 16th IEEE Symposium on Logic in Computer Science (LICS 2001), pages 421-130, 2001.

2. N. Alon, T. Milo, F. Neven, D. Suciu, and V. Vianu. XML with data values: Typechecking revisited. In Proc. 20th Symposium on Principles of Database Systems (PODS 2001), pages 560-572, 2001.

3. G. J. Bex, S. Maneth, and F. Neven. A formal model for an expressive fragment of XSLT. Information Systems, 27(1):21-39, 2002.

4. A. Brüggemann-Klein, M. Murata, and D. Wood. Regular tree and regular hedge languages over unranked alphabets: Version 1, april 3, 2001. Technical Report HKUST-TCSC-2001-0, The Hongkong University of Science and Technology, 2001.

5. P. Buneman, M. Fernandez, and D. Suciu. UnQ1: a query language and algebra for semistructured data based on structural recursion. The VLDB Journal, 9(1):76$110,2000$.

6. James Clark. XSL transformations version 1.0. http://www.w3.org/TR/WD-xslt, august 1999.

7. World Wide Web Consortium. XML Schema. http://www.w3.org/XML/Schema.

8. S.A. Cook. An observation on time-storage trade-off. Journal of Computer and System Sciences, 9(3):308-316, 1974.

9. J. Engelfriet and H. Vogler. Macro tree transducers. Journal of Computer and System Sciences, 1985. 
10. M.R. Garey and D.S. Johnson. Computers and Intractability: A Guide to the Theory of NP-Completeness. Freeman, 1979.

11. F. Gécseg and M. Steinby. Tree languages. In G. Rozenberg and A. Salomaa, editors, Handbook of Formal Languages, volume 3, chapter 1, pages 1-68. Springer, 1997.

12. R. E. Ladner, R. J. Lipton, and L. J. Stockmeyer. Alternating pushdown and stack automata. SIAM Journal on Computing, 13(1):135-155, 1984.

13. S. Maneth and F. Neven. Structured document transformations based on XSL. In R. Connor and A. Mendelzon, editors, Research Issues in Structured and Semistructured Database Programming (DBPL'99), volume 1949 of Lecture Notes in Computer Science, pages 79-96. Springer, 2000.

14. T. Milo and D. Suciu. Type inference for queries on semistructured data. In Proceedings of the Eighteenth ACM Symposium on Principles of Database Systems, pages 215-226. ACM Press, 1999.

15. T. Milo, D. Suciu, and V. Vianu. Type checking for XML transformers. In Proceedings of the Nineteenth ACM Symposium on Principles of Database Systems, pages 11-22. ACM Press, 2000.

16. F. Neven. Automata theory for XML researchers. SIGMOD Record, 31(3), 2002.

17. F. Neven and T. Schwentick. XML schemas without order. Unpublished manuscript, 1999.

18. F. Neven and T. Schwentick. Query automata on finite trees. Theoretical Computer Science, 275:633-674, 2002.

19. Y. Papakonstantinou and V. Vianu. DTD inference for views of XML data. In Proc. 20th Symposium on Principles of Database Systems (PODS 2001), pages 35-46. ACM Press, 2001.

20. H. Seidl. Deciding equivalence of finite tree automata. SIAM Journal on Computing, 19(3):424-437, 1990.

21. H. Seidl. Haskell overloading is DEXPTIME-complete. Information Processing Letters, 52(2):57-60, 1994.

22. L. J. Stockmeyer and A. R. Meyer. Word problems requiring exponential time: Preliminary report. In Conference Record of Fifth Annual ACM Symposium on Theory of Computing, pages 1-9, Austin, Texas, 30 April-2 May 1973.

23. D. Suciu. Typechecking for semistructured data. In Proceedings of the 8th Workshop on Data Bases and Programming Languages (DBPL 2001), 2001.

24. D. Suciu. The XML typechecking problem. SIGMOD Record, 31(1):89-96, 2002.

25. A. Tozawa. Towards static type checking for XSLT. In Proceedings of ACM Symposium on Document Engineering, 2001.

26. M. Y. Vardi. A note on the reduction of two-way automata to one-way automata. Information Processing Letters, 30:261-264, March 1989. 